\title{
Application of an expectation maximization method to the reconstruction of X-ray-tube spectra from
}

\author{
M. Endrizzi ${ }^{\mathrm{a}, *}$, P. Delogu ${ }^{\mathrm{b}}$, P. Oliva ${ }^{\mathrm{c}}$ \\ ${ }^{a}$ Dipartimento di Fisica, Università di Siena, Via Roma 56, 53100 and INFN, Sezione di \\ Pisa, 56127 Pisa, Italy \\ ${ }^{b}$ Dipartimento di Fisica "E. Fermi", Università di Pisa, Largo B. Pontecorvo 3, 56127 \\ Pisa and INFN, Sezione di Pisa, 56127 Pisa, Italy \\ $9 \quad{ }^{c}$ Struttura Dipartimentale di Matematica e Fisica, Università di Sassari, Via Vienna 2, \\ 07100 Sassari, Italy and INFN, Sezione di Cagliari, 09042 Cagliari, Italy
}

\section{Abstract}

An expectation maximization method is applied to the reconstruction of $\mathrm{X}$-ray tube spectra from transmission measurements in the energy range $7-40 \mathrm{keV}$. A semiconductor single-photon counting detector, ionization chambers and a scintillator-based detector are used for the experimental measurement of the transmission. The number of iterations required to reach an approximate solution is estimated on the basis of the measurement error, according to the discrepancy principle. The effectiveness of the stopping rule is studied on simulated data and validated with experiments. The quality of the reconstruction depends on the information available on the source itself and the possibility to add this knowledge to the solution process is investigated. The method can produce good approximations provided that the amount of noise in the data can be estimated.

\section{Introduction}

$\mathrm{X}$-ray-spectrum reconstruction from transmission data is an ill-posed problem that aims to the calculation of the energy distribution of the radiation by using a series of attenuated intensity measurements. An intensity measurement performed by a detector is generally the result of integration over the energy distribution of the radiation. Information about this distribution is recovered by using a series of attenuation measurements in which the

${ }^{*}$ Current address: Department of Medical Physics and Bioengineering, University College London, Malet Place, Gower Street, Lołdon WC1E 6BT, UK

Email address: m.endrizzi@ucl.ac.uk (M. Endrizzi) 
spectrum is progressively modified by varying the filtration. A number of different approaches have been proposed for the solution of this problem, including the use of the Laplace transform, least squares methods, singular value decomposition and iterative approaches [1-20]. In this work we focus on the application to experimental data of the expectation-maximization method $[14,17]$ to iteratively solve the ill-conditioned linear system that relates the spectral distribution of the X-ray beam to its transmission. With this method we investigate a relatively low-energy range with the use of the rithm. The minimum knowledge that is required for applying this criterion is the noise level present in the experimental data. Using simulation studies number of iterations to be performed. If further details about the source are known, this information can be inserted in the solution process by conditioning the starting point of the algorithm. The influence of this conditioning on the solution is studied and evaluated through computer simulations and experiments. The possibility of measuring the transmission in different ways is experimentally explored using various types of radiation detectors: photon counter, ionization chamber and imaging detector.

\section{Materials and Methods}

The total signal revealed by the detector $\mathcal{T}(t)$, as a function of the thickness $t$ of the attenuator material, can be related to the spectrum $\mathcal{X}(E)$ of the X-ray source as

$$
\mathcal{T}(t)=\int_{0}^{\infty} \mathcal{S}(E) e^{-\mu(E) t} d E
$$

24 $E$ is the energy, $\mu(E)$ is the linear attenuation coefficient of the attenuator and $\int \mathcal{X}(E) d E=1$. After discretization, Eq. 1 can be written as $T=A S$ $T$ and $S$ are vectors and $A$ is a matrix. $T_{m}$ (with $m=1 \ldots M$ ) is the measurement with the $m$-th thickness of the attenuator material, while $S_{n}$ (with $\left.n=1 \ldots N\right)$ is the contribution of the $n$-th energy bin of the spectrum, weighted with the detector response $D_{n}$. The matrix $A_{m n}=e^{-\mu_{n} t_{m}}$ contains the properties of the attenuator at the energy corresponding to the $n$-th energy bin and for the thickness used in the $m$-th transmission measurement. 
Once the transmission curve $T$ has been measured, we iteratively apply the set of equations $[14,17,22,23]$

$$
\left\{\begin{array}{l}
S_{n}^{k+1}=S_{n}^{k} f_{n}^{k} \\
f_{n}^{k}=\frac{\sum_{m} A^{t}{ }_{n m} \frac{T_{m}}{\sum_{n^{\prime}} A_{m n^{\prime}} S_{n^{\prime}}^{k}}}{\sum_{m} A^{t}{ }_{n m}}
\end{array}\right.
$$

3 where $S_{n}^{k+1}$ is the $n$-th energy bin after $(k+1)$ iterations, $T_{m}$ is the $m$-th measurement of the transmission curve and $A^{t}$ is the transpose of the matrix $A$. Each solution $S^{k}$ is computed based on the vector of attenuation 6 measurements $T$, the matrix $A$ and the output of the previous cycle. The algorithm requires an initial guess $S^{0}$ and a stopping rule to break the loop. To simulate a situation in which no information about the source is available, 9 we start the iteration from a flat distribution. To impose a constraint on the maximum energy of the spectrum, we start the iteration from a triangleshaped distribution that falls to zero at $E_{\max }$. If the material composing the 12 anode is known, the iteration can start from a larger value for the energy bins expected to contain characteristic emissions. The relative intensities between the energy bins containing characteristic emissions and the others 15 are not easily predicted; they depend on the tube voltage and on a number of parameters related to the design of the source [24]. Based on experience, we set these values to be three times the value of the background. This was 18 chosen by looking at calculated spectra, using the catalog provided by The Institute of Physics and Engineering in Medicine [25] (IPEM), in conditions similar to those encountered in the experiments. A further refinement of the starting point can be done taking into account the inherent filtration of the X-ray source.

Considering the measured transmission curve as $T=T^{e x}+e$, with $T^{e x}$ representing the exact transmission curve and $e$ the noise related to the measurement process, the discrepancy principle provides the inequality

$$
\left\|T-A S^{k}\right\| \leq\|e\|
$$

where $\|e\|=\sqrt{\sum_{i=1}^{N} e_{i}{ }^{2}}$. This can be used to estimate the number of 27 iterations to be performed: when the left term in Eq. 3 (residual) becomes equal to or smaller than the norm of the noise the iteration is stopped. In the experiments, $\|e\|$ can be estimated using the experimental errors on 30 the actual transmission measurements. Other two parameters, that can be 
Table 1: Instrumentation and materials used in the experiments

\begin{tabular}{lr}
\hline X-ray tubes & microfocus, tungsten target \\
Hamamatsu 60KVMFX-SPL & $\begin{array}{r}\text { rotating anode, tungsten target } \\
\text { Gilardoni Rotagil }\end{array}$ \\
Gilardoni Sylvia & rotating anode, molybdenum target \\
\hline Detectors & photon counter, energy resolving \\
Amptek, XR-100T-CZT & ionisation chamber and meter \\
PTW 34047, PTW Unidos E & ionisation chamber and meter \\
Radcal 20X6-6, Radcal 2026C & active pixel CMOS sensor \\
Hamamatsu C9728DK-10 & \\
\hline Filters & high-purity aluminum (99.999\%) \\
Good Fellow filters
\end{tabular}

considered when comparing the reconstructed spectrum to the reference one, are the mean energy $m_{1}=\int_{0}^{\infty} E \mathcal{X}(E) d E$ and the energy spread $m_{2}{ }^{2}=$ $3 \int_{0}^{\infty}\left(E-m_{1}\right)^{2} \mathcal{X}(E) d E$.

\subsection{Simulations}

The convergence properties of the method are evaluated by means of nu6 merical simulations: the attenuation of the X-ray beam is calculated starting from a known spectrum $X^{e x}$ and the noise is numerically added to the exact transmission. From this simulated data, the spectrum is iteratively calcu9 lated using Eq. 2 and $d(k)=\left\|X^{k}-X^{e x}\right\|$ is computed after each iteration $k$. The simulations are carried out as follows. An X-ray spectrum $X(E)$ is computed for a tungsten target at $40 \mathrm{kV}$ and $2.5 \mathrm{~mm}$ of aluminum inher12 ent filtration (IPEM). A set of 22 attenuation measurements is computed with a minimum intensity $\left(T_{M} / T_{1}\right)$ of about $10^{-2}$. The exact transmission curve $T^{e x}$ is perturbed to simulate the measurement noise. Three different noise intensities are considered and the perturbation vector $e$ is randomly extracted from a normal distribution with 0 mean and standard deviations of $10^{-3}, 5 \times 10^{-3}$ and $10^{-2}$ times the value of the measurement. The perturbed simulated transmission curve is then $T=T^{e x}+e$.

\subsection{Experimental}

The single-photon-counting measurement has been experimentally realized using a microfocus source (Hamamatsu, 60KVMFX-SPL) at 10 and 15 $\mathrm{kV}$, with $200 \mu \mathrm{A}$. A $0.5 \mathrm{~mm}$ aluminum filter is placed close to the tube 
focus (Fig. 1a). The detector is a CZT semiconductor device (Amptek, XR-100T-CZT). In all the experiments we use high-purity aluminum filters 3 (Good Fellow, $99.999 \%$ purity) as attenuators and an energy-bin width of a fraction of a keV (half or quarter) to appropriately reproduce the spectra in the energy range under investigation. The transmission curve is measured 6 by counting the photons that have been detected by the detector. A series of NIM (Nuclear Instrumentation Module) modules is used to discriminate the noise and count the events. The chain was composed by a polarity inthis semiconductor device, it is also possible to measure the energy of each interacting photon directly.

12 For measurements with an ionization chamber, $\mathcal{D}(E)=\left(\frac{\mu_{e n}(E)}{\rho}\right)_{\text {air }}\left(\frac{e_{c}}{\bar{W}}\right)_{\text {air }} E$, with $\mu_{e n}$ the energy absorption coefficient of air, $\rho$ the density $\left(\mathrm{g} / \mathrm{cm}^{3}\right), \bar{W}$ is the mean energy expended per ion pair formed in a gas, $e_{c}$ is the charge meas measurement is a conventional tube for X-ray diagnostic imaging (Gilardoni Rotagil) with a tungsten anode and $2.5 \mathrm{~mm}$ aluminum of inherent filtration, tion chambers (PTW 34047 and dosimeter PTW Unidos E; Radcal 20X6-6 and dosemeter Radcal 2026C) for a series of aluminum thicknesses ranging can be corrected by using the ratio of the readings of the two ionization chambers arranged as depicted in Fig. $1 b$.

$24 \quad$ Experimental data are collected also using a characterized [27] active pixel CMOS sensor (Hamamatsu C9728DK-10). The intensity is averaged over an area of about $100 \times 100$ pixels. In principle, a transmission curve can be measured pixel-by-pixel, and a two-dimensional distribution of spectra can be computed for simultaneously characterizing a non-uniform X-ray beam $[28,29]$. The experimental set-up is the same as reported in Fig. 1b except 30 for the replacement of IC 2 with the flat panel. The source is a molybdenumanode X-ray tube (Gilardoni Sylvia) operated at $28 \mathrm{kV}$ and $20 \mathrm{~mA}$, with an inherent filtration of $1 \mathrm{~mm}$ of beryllium and $30 \mu \mathrm{m}$ of molybdenum. The з3 aluminum filter thicknesses range between 0 and $6 \mathrm{~mm}$. All the materialspecific coefficients used in these calculations were obtained from the xraylib library $[30,31]$. 


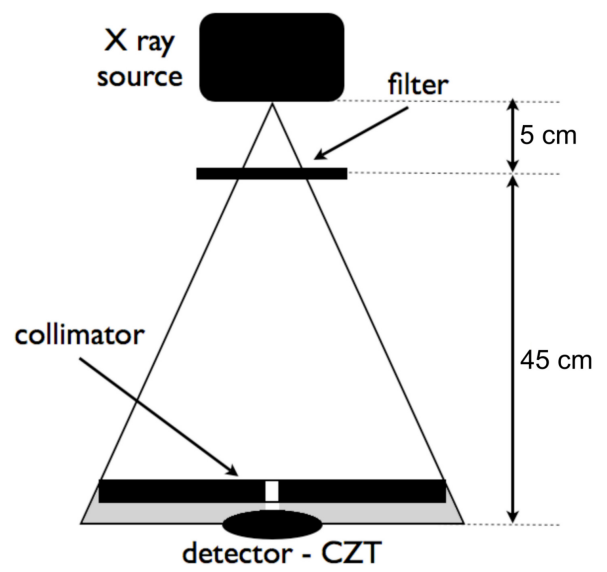

(a)

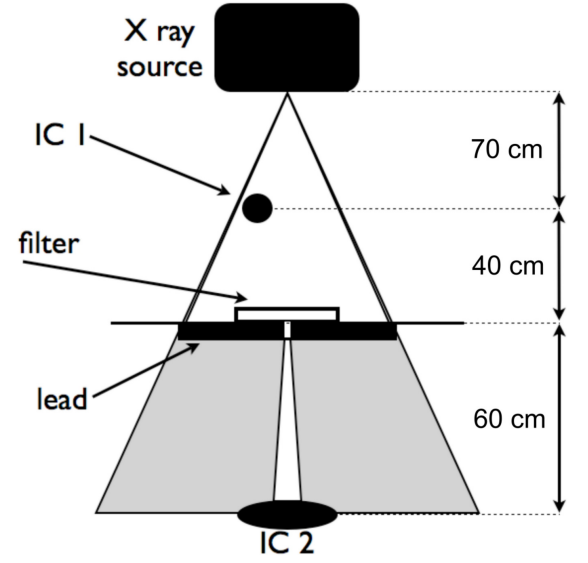

(b)

Figure 1: (a) single-photon-counting experimental set-up, the collimator has a diameter of $400 \mu \mathrm{m}$. (b) ionization-chamber measurement set-up, the collimator has a diameter of $1 \mathrm{~cm}$.

\section{Results and Discussion}

The reconstructions obtained from the simulated transmission curves (Section 2.1) are reported in Fig. 2. Each reconstruction takes a few minutes on a $2.33 \mathrm{GHz} \mathrm{CPU}$. The ordinate of all the spectra plots is such that the sum over all the energy bins is normalized to unity. The optimum approximation (when $d(k)$ is minimum) can be compared with the solutions selected using the discrepancy principle. The noise intensity is $1 \%$. The mean energy of the spectrum obtained using the stopping rule is reconstructed with less than $1 \%$ error and the energy spread with approximately $1 \%$ error in Fig. 2b and with a $20 \%$ error in Fig. 2a. In Fig. 2a the iteration starts from a flat distribution and the reference spectrum is approximated with a smooth curve. In Fig. 2b the reconstruction is done imposing $E_{\max }$, the result is almost superimposed on the reference spectra. By looking at the semi-convergence graphs (Fig. 3) it can be seen that the minimum of $d(k)$ depends more on the starting point than on the noise level: lower minima are reached in the plots in Fig. $2 \mathrm{~b}$ and relatively smaller differences are observed between the different noise cases. Test were performed with 5\% noise but they were not as well reproducible as for the other noise intensities considered here: we found some cases in which Eq. 3 was not satisfied. The 


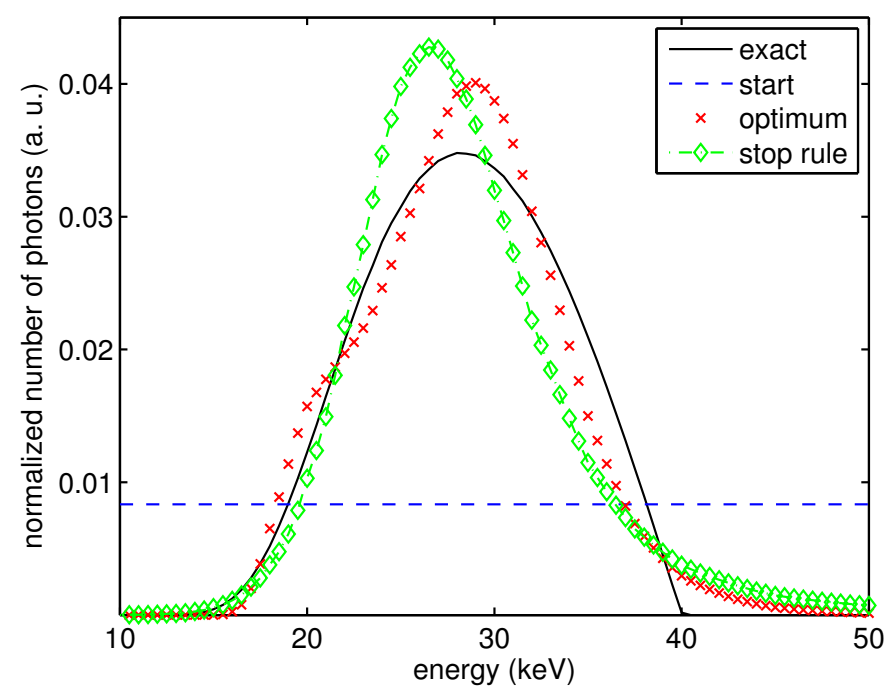

(a)

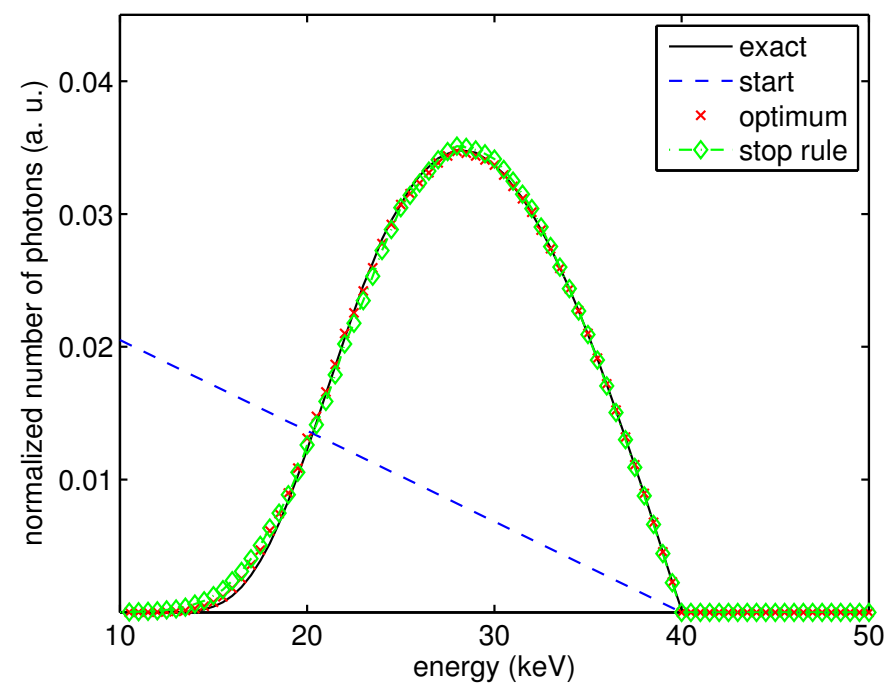

(b)

Figure 2: Simulated data. Tungsten-target X-ray tube at $40 \mathrm{kV}$ with $2.5 \mathrm{~mm}$ of aluminum as inherent filtration. The optimum approximation and the one selected using the stopping rule are compared. The initial distribution is flat (a) or triangle-shaped (b). 
optimum values found for $k$ are $k_{\text {opt }}=\{229179,37819,11458\}$ in the case of flat input distribution, while the discrepancy principle, i.e. when the inequal3 ity Eq. 3 is satisfied, suggests $k_{t h}=\{7265,2843,318\}$, respectively, for the $10^{-3}, 5 \times 10^{-3}$ and $10^{-2}$ noise cases. For the triangle-shaped input, the sets of numbers are $k_{\text {opt }}=\{5020,6467,1203\}$ and $k_{t h}=\{1469,637,600\}$. The 6 values estimated by using the discrepancy principle are smaller than those indicated by a minimum $d(k)$, however it can be seen from the graphs in Fig. 3 that the actual difference (in terms of $d(k)$ ) is relatively small in the two cases. This is also reflected in the actual distributions (Fig. 2) which are close one to each other. To test the algorithm on multiple noise realizations, the exact transmission curve is perturbed $10^{3}$ times, for the case with $1 \%$ noise. Each energy bin $n$ has now a distribution of values, each one coming from a different noise realization, from which the mean $\mu_{n}$ and the standard deviation $\sigma_{n}$ are extracted. The graphs $\mu_{n}+\sigma_{n}$ and $\mu_{n}-\sigma_{n}$ are plotted in of the spectrum in each bin, suggesting that the results are reproducible.

The tungsten anode spectra at 10 and $15 \mathrm{kV}$, reconstructed from the single-photon-counting detector measurements (Tab. 1 line 2 and 6), are shown in Fig. 5. In both cases, the error is less than $0.5 \%$ for the mean energy and less than $2 \%$ for the spread. The reconstructions are fairly close, 21 in terms of mean energy and energy spread, to the reference spectra measured using the semiconductor device with a multi-channel analyzer [32]. We note that the spectrum at $15 \mathrm{kV}$ is reproduced with less accuracy with respect to 24 the one at $10 \mathrm{kV}$; this might be due to the stronger line emissions which are not well approximated by using a smooth curve.

The tungsten anode spectrum at $40 \mathrm{kV}(2.5 \mathrm{~mm}$ of aluminum inherent filtration) is reconstructed from ionization chambers measurements (Fig. 6 , Tab. 1 line 3, 7 and 8). Imposing $E_{\max }$ leads to a solution that is almost superimposed on the reference spectrum (IPEM). The reference mean energy ues are 28.96 and $7.44 \mathrm{keV}$ starting from a flat spectrum and 28.16 and 5.16 $\mathrm{keV}$ imposing $E_{\max }$. Starting the iteration from a flat distribution consisThis could be due to the fact that the algorithm updates each bin by multiplication and therefore those bins initially set to non-zero values will always be non-zero. Another contribution could be related to the behaviour of the attenuation as function of energy: at higher energies the relative change in attenuation becomes smaller and this makes harder X-rays more difficult to 


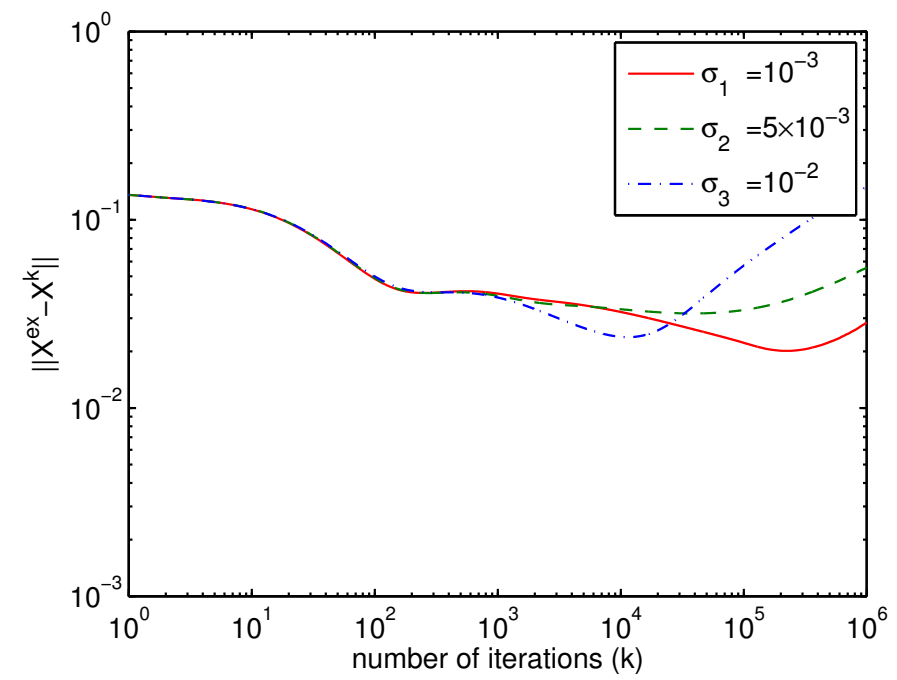

(a)

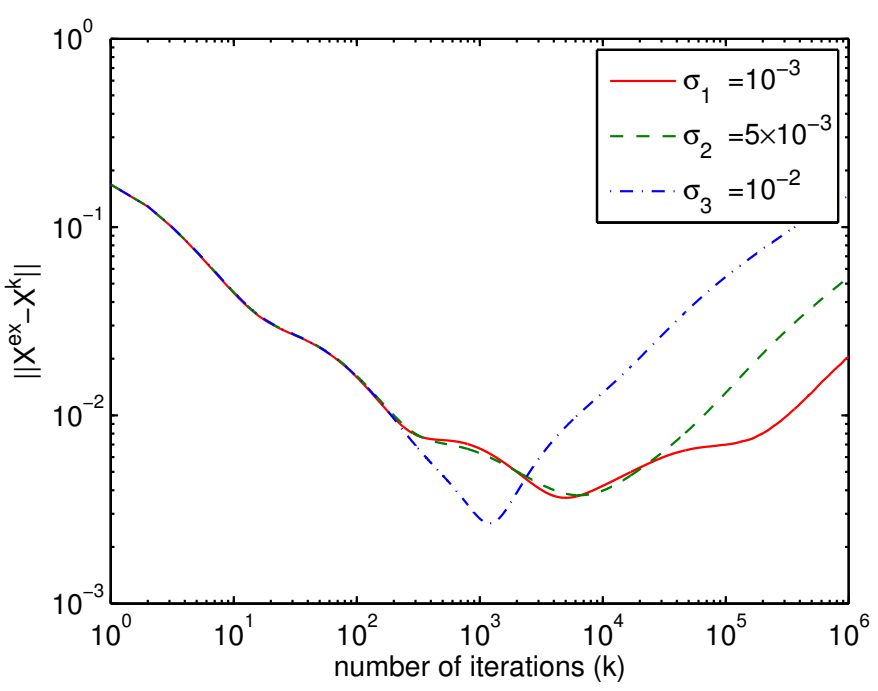

(b)

Figure 3: Semi-convergence for the reconstruction of tungsten target, $40 \mathrm{kV}$ spectrum (Fig. 2). (a) flat initial distribution and (b) triangle-shaped input. 


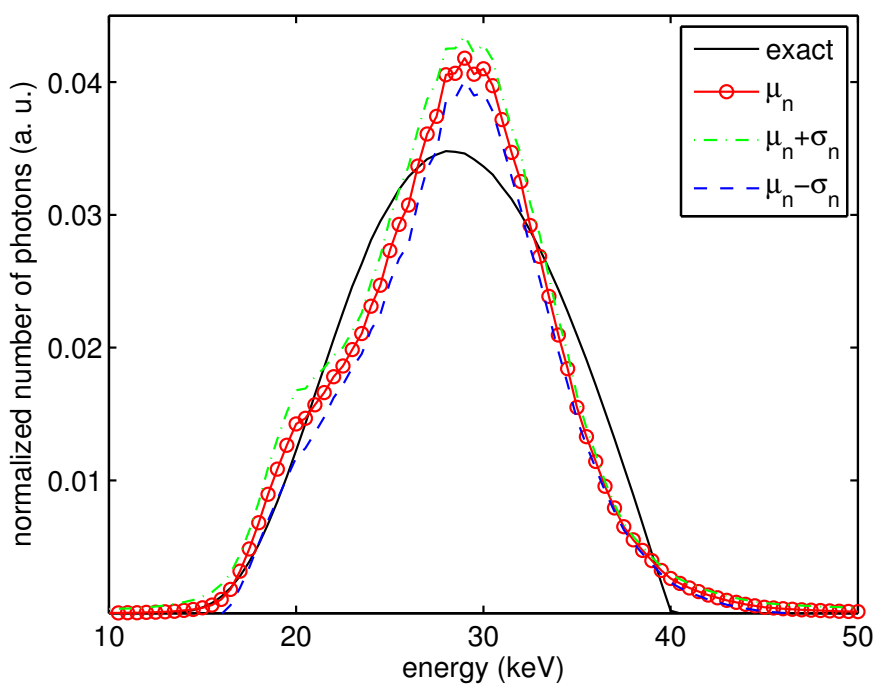

(a)

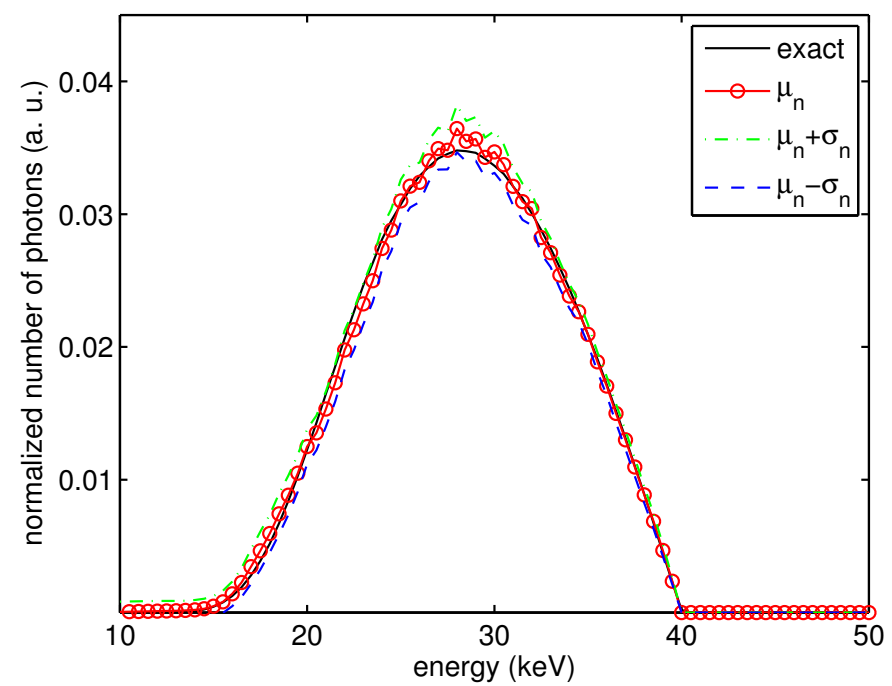

(b)

Figure 4: Simulation with multiple noise (1\%) realizations for the tungsten target $40 \mathrm{kV}$ spectrum, using (a) a flat starting point and (b) imposing $E_{\max }$.

be discriminated by the algorithm.

The molybdenum-anode spectra, reconstructed using the flat-panel sen3 sor data (Tab. 1 line 4 and 9), are shown in Fig. 7. The mean energy is estimated with about $3 \%$ error for each starting point, and the energy spread 


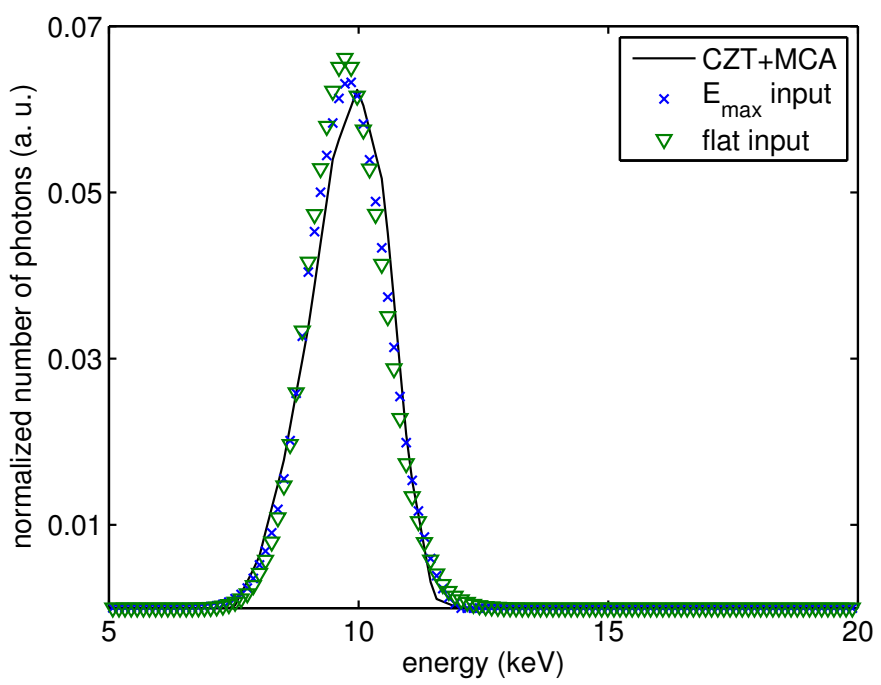

(a)

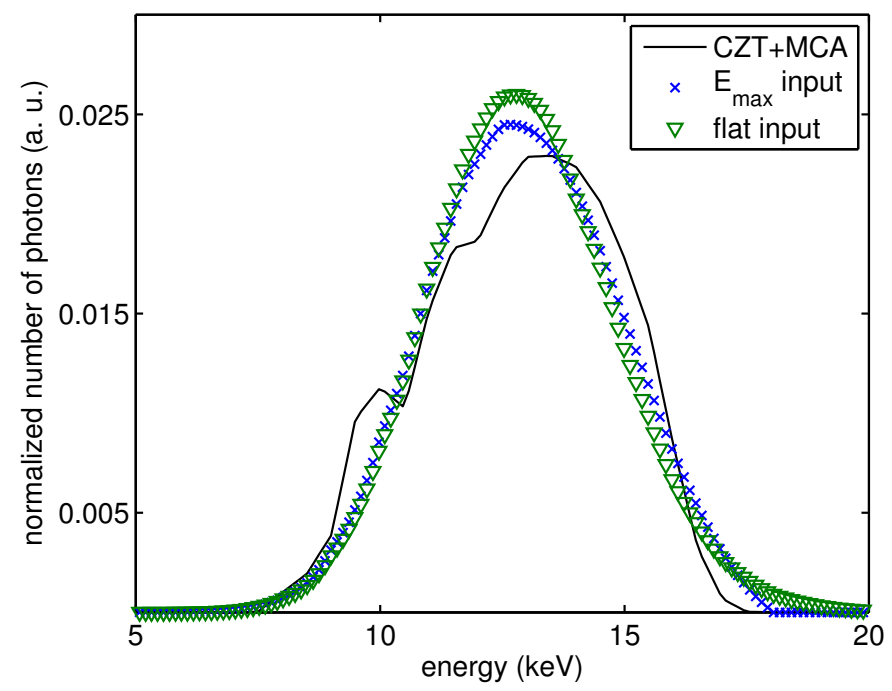

(b)

Figure 5: Spectra reconstructions from photon counts (Tab. 1 line 2 and 6). (a) tungsten anode operated at $10 \mathrm{kV}$ and (b) tungsten anode at $15 \mathrm{kV}$. The inherent filtration is 0.5 $\mathrm{mm}$ of aluminum in both cases. 


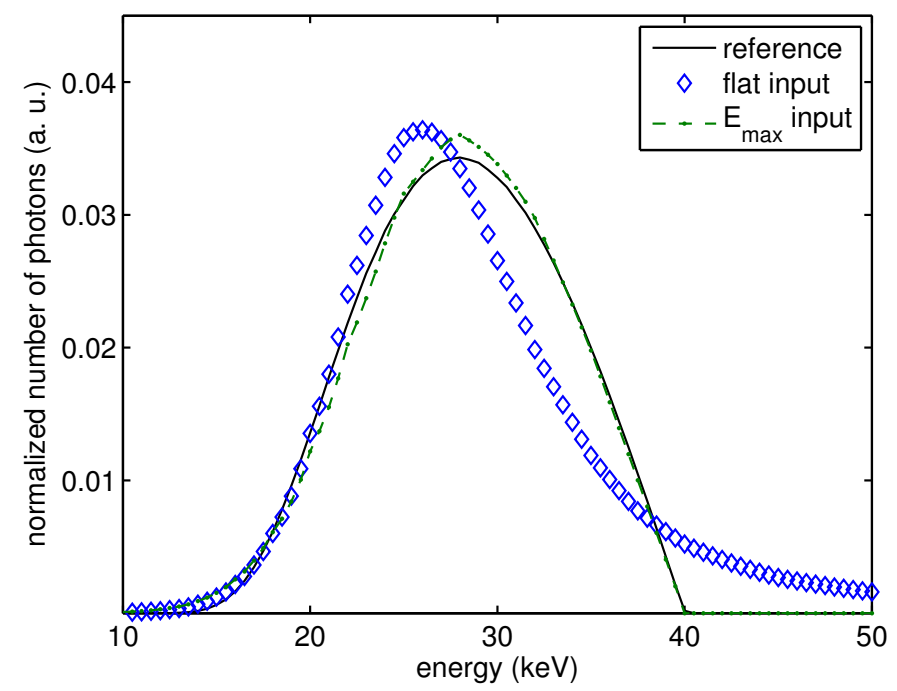

Figure 6: Spectrum reconstructions from experimental measurements made with ionization chambers (Tab. 1 line 3, 7 and 8), tungsten target at $40 \mathrm{kV}$.

is reconstructed within $2 \%$ error. Note that the reconstructed spectra are truncated for energies below $7 \mathrm{keV}$ because of the negligible sensitivity of the detector in this energy range. Truncating at $7 \mathrm{keV}$ does not strongly affect the spectrum reconstruction because we are discarding a very small fraction $\left(1 \times 10^{-4}\right)$ of photons. The reference spectrum is measured with the portable 6 semiconductor (CZT) device previously used and was also computed using the catalog from IPEM. Three different initial distributions are used: flat, triangle-shaped with discontinuities at molybdenum characteristic emissions 9 and triangle-shaped with discontinuities plus filtration. The filtering is computed considering that the X-ray tube has an inherent filtration of $30 \mu \mathrm{m}$ of molybdenum plus $1 \mathrm{~mm}$ of beryllium, as stated by the technical specification of the machine. This type of spectrum is a standard for mammography, the quality of the reconstruction can be evaluated also by calculating the mean glandular dose, which is a quantity of interest in clinical applications [33].

15 The mean glandular dose is computed for each X-ray spectra and normalized to unit fluence. The data, together with the percent deviation with respect to the reference value, are reported in Tab. 2. The difference between the reconstructed and the reference spectra is approximately $5 \%$. 


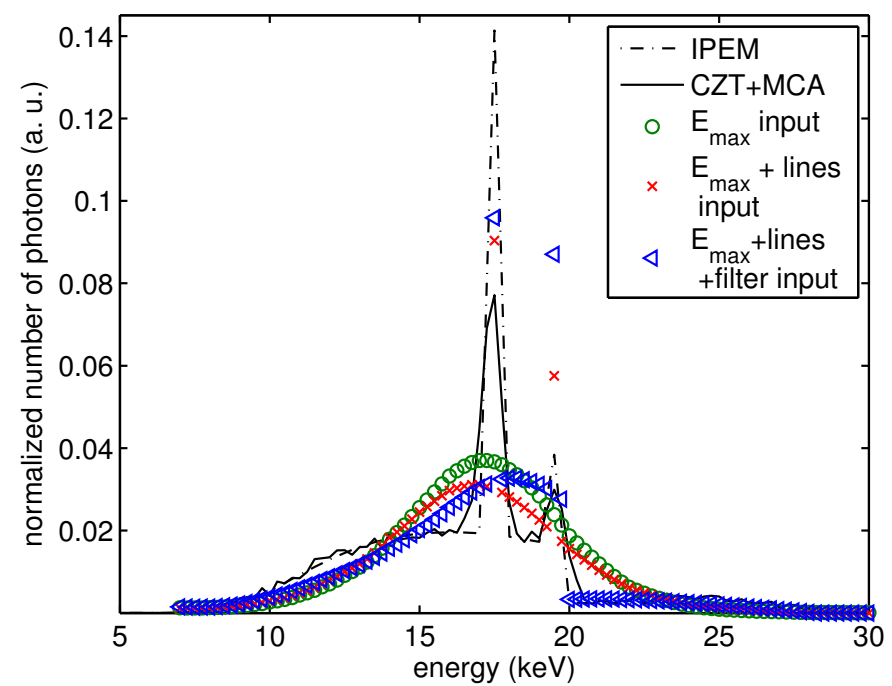

Figure 7: Typical mammography spectrum reconstruction in which the transmission curve was measured using an imaging detector (Tab. 1 line 4 and 9).

\begin{tabular}{cccccc}
\hline & $\begin{array}{c}\text { IPEM catalog } \\
\text { spectrum }\end{array}$ & $\begin{array}{c}\text { CZT measured } \\
\text { spectrum }\end{array}$ & $\begin{array}{c}\text { Flat } \\
\text { starting point }\end{array}$ & $\begin{array}{c}E_{\max } \text { and lines } \\
\text { starting point }\end{array}$ & $\begin{array}{c}E_{\max }, \text { lines and filtration } \\
\text { starting point }\end{array}$ \\
\hline$\left(10^{-9}\right) \times$ & 53.91 & $53.68(<0.5 \%)$ & $56.82(5.4 \%)$ & $56.61(5.0 \%)$ & $56.30(4.4 \%)$ \\
\hline
\end{tabular}

Table 2: Mean glandular dose comparison for the molybdenum anode at $28 \mathrm{kV}$ spectrum. The dose is computed for unit photon fluence $\left(\mathrm{mGy} \times \mathrm{mm}^{2}\right)$. 


\section{Conclusions}

An expectation-maximization algorithm has been used with the discrepancy principle as a method for X-ray-spectrum reconstruction from attenuation data. The transmission curve has been measured using different physical quantities, various qualities of X-ray beams and experimental conditions.

6 The method has been tested on simulated data and validated with experiments using conventional X-ray tubes in the $7-40 \mathrm{keV}$ energy range. With few percent noise, the discrepancy principle is a suitable criterion to select results with experimental data in terms of mean energy, energy spread and shape of the distribution. Refinement has been evaluated on the basis of the information available. The minimum knowledge required is the noise in the transmission curve. If further details about the X-ray source are known (maximum energy, characteristic emissions, inherent filtration), the reconstruction can be improved by preconditioning the initial spectrum. Those additional constraints become important when the spectrum contains sharp features (e.g. mammography spectrum). If the distribution changes smoothly, the reconstructed spectrum is usually very close to the exact one (e.g. filtered tungsten spectra). Mean energy and the energy spread are usually accurately estimated, even when the shape of the spectrum is not precisely reproduced. Finally, an evaluation based on the computation of the mean glandular dose provided about 5\% agreement between the reference spectrum and the one reconstructed with the proposed method.

24 [1] L. Silberstein, Determination of the spectral composition of X-ray radiation from filtration data, J. Opt. Soc. Am. 22 (5) (1932) 265-278.

[2] J. R. Greening, The determination of x-ray wavelength distributions from absorption data, Proceedings of the Physical Society. Section A 63 (11) (1950) 1227.

[3] P. M. Joseph, Mathematical method for determining kvp from x-ray attenuation measurements, Medical Physics 2 (4) (1975) 201-207.

[4] L. C. Baird, X-ray spectra vs attenuation data: A theoretical analysis, Medical Physics 8 (3) (1981) 319-323.

33 [5] P. H. Huang, K. R. Kase, B. E. Bjaerngard, Spectral characterization of $4 \mathrm{mv}$ bremsstrahlung by attenuation analysis, Medical Physics 8 (3) (1981) 368-374. 
[6] S. Tominaga, The estimation of x-ray spectral distributions from attenuation data by means of iterative computation, Nuclear Instruments and Methods in Physics Research 192 (23) (1982) 415 - 421.

[7] H. Kramer, H. V. Seggern, The determination of x-ray spectra from attenuation data: Part i: The potentials of various methods, Nuclear Instruments and Methods in Physics Research 213 (23) (1983) 373 380.

[8] P. Francois, A. Catala, C. Scouarnec, Simulation of x ray spectral reconstruction from transmission data by direct resolution of the numeric system af=t, Medical Physics 20 (6) (1993) 1695-1703.

[9] M. Krmar, J. Slivka, I. Bikit, M. Veskovic, L. Conkic, Evaluation of bremsstrahlung spectra generated by a 4 mev linear accelerator, Medical Physics 23 (5) (1996) 651-654.

[10] R. G. Waggener, M. M. Blough, J. A. Terry, D. Chen, N. E. Lee, S. Zhang, W. D. McDavid, X-ray spectra estimation using attenuation measurements from $25 \mathrm{kvp}$ to $18 \mathrm{mv}$, Medical Physics 26 (7) (1999) $1269-1278$.

18 [11] M. Stampanoni, M. Fix, P. Francois, P. Ruegsegger, Computer algebra for x-ray spectral reconstruction between 6 and 25 mv, Medical Physics 28 (3) (2001) 325-327.

[12] W. H. Hinson, J. D. Bourland, Spectral reconstruction of high energy photon beams for kernel based dose calculations, Medical Physics 29 (8) (2002) 1789-1796.

[13] B. Armbruster, R. J. Hamilton, A. K. Kuehl, Spectrum reconstruction from dose measurements as a linear inverse problem, Physics in Medicine and Biology 49 (22) (2004) 5087.

[14] E. Y. Sidky, L. Yu, X. Pan, Y. Zou, M. Vannier, A robust method of $\mathrm{X}$-ray source spectrum estimation from transmission measurements: Demonstrated on computer simulated, scatter-free transmission data, Journal of Applied Physics 97 (12) (2005) 124701. 
[15] L. D. Paniak, P. M. Charland, Enhanced bremsstrahlung spectrum reconstruction from depthdose gradients, Physics in Medicine and Biology 50 (14) (2005) 3245.

[16] V. Delgado, Determination of x-ray spectra from attenuation data by imposing a priori positiveness and bounded support: Theory and exper-

$6 \quad$ imental validation, Med. Phys. 34 (3) (2007) 994-1006.

[17] L. Zhang, G. Zhang, Z. Chen, Y. Xing, J. Cheng, Y. Xiao, X-ray spectrum estimation from transmission measurements using the expecta-

9 tion maximization method, in: Nuclear Science Symposium Conference Record, 2007. NSS '07. IEEE, Vol. 4, 2007, pp. 3089-3093.

[18] V. Delgado, Determination of x-ray spectra from al attenuation data by imposing a priori physical features of the spectrum: Theory and experimental validation, Med. Phys. 36 (1) (2009) 142-148.

[19] M. Manciu, F. Manciu, T. Vulcan, E. Nes, R. Waggener, Robust megavoltage x-ray spectra estimation from transmission measurements., J. Xray. Sci. Technol. 17 (1) (2009) 85-99.

[20] X. Duan, J. Wang, L. Yu, S. Leng, C. H. McCollough, Med. Phys. 38 (2) (2011) 993-997.

[21] V. A. Morozov, On the solution of functional equations by the method of regularization, Sov. Math. Dokl. 7 (1966) 414-417.

[22] Y. Vardi, D. Lee, From image deblurring to optimal investments: Maximum likelihood solutions for positive linear inverse problems, J. R. Statistic. Soc. B 55 (3) (1993) 569-612.

[23] M. Bertero, P. Boccacci, Introduction to inverse problems in imaging, Institute of Physics Publishing, 1998.

[24] A. G. Michette, C. J. Buckley, X-Ray Science and Technology, Institute of Physics Publishing, 1993.

[25] K. Cranley, B. Gilmore, G. Fogarty, L. Desponds, Catalogue of diagnostic x-ray spectra and other data, IPEM Report 78 (CD-Rom Edition (Electronic version prepared by D. Sutton), York: The Institute of Physics and Engineering in Medicine (IPEM), 1997. 
[26] F. H. Attix, Introduction to Radiological Physics and Radiation Dosimetry, Wiley - New York, 1986.

3 [27] M. Endrizzi, P. Oliva, B. Golosio, P. Delogu, CMOS APS detector characterization for quantitative X-ray imaging, Nuclear Instruments and Methods in Physics Research A 703 (2013) 26-32.

6 [28] M. Endrizzi, P. Delogu, A. Stefanini, X-ray spectra reconstruction from analysis of attenuation data: A Back Scattering Thomson source application, Nuclear Instruments and Methods in Physics Research A 608 $9 \quad$ (2009) 78 .

[29] B. Golosio, M. Endrizzi, P. Oliva, P. Delogu, M. Carpinelli, I. Pogorelsky, V. Yakimenko, Measurement of an inverse compton scattering source

$12 \quad$ local spectrum using k-edge filters, Applied Physics Letters 100 (16) (2012) 164104.

[30] A. Brunetti, M. S. del Rio, B. Golosio, A. Simionovici, A. Somogyi, A library for x-raymatter interaction cross sections for x-ray fluorescence applications, Spectrochimica Acta Part B: Atomic Spectroscopy 59 (1011) (2004) 1725 - 1731, 17th International Congress on X-Ray Optics and Microanalysis.

[31] T. Schoonjans, A. Brunetti, B. Golosio, M. S. del Rio, V. A. Sol, C. Ferrero, L. Vincze, The xraylib library for x-raymatter interactions. recent developments, Spectrochimica Acta Part B: Atomic Spectroscopy 66 (1112) (2011) $776-784$.

[32] S. Stumbo, U. Bottigli, B. Golosio, P. Oliva, S. Tangaro, Direct analysis of molybdenum target generated x-ray spectra with a portable device, Med. Phys. 31 (10) (2004) 2763-2770.

[33] G. Hammerstein, D. Miller, D. White, M. Masterson, H. Woodard, J. Laughlin, Absorbed radiation dose in mammography, Radiology 130 (2) (1979) 485-91. 